\title{
Numerical Rate Equation Approach to Picosecond Charge State Dynamics in CdTe/ZnTe Quantum Dots
}

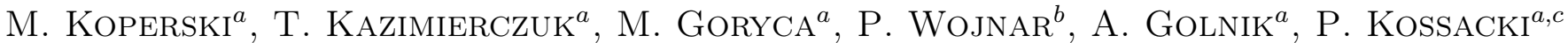 \\ AND J.A. GAJ ${ }^{a}$ \\ ${ }^{a}$ Institute of Experimental Physics, University of Warsaw, Hoża 69, 00-681 Warsaw, Poland \\ ${ }^{b}$ Institute of Physics, Polish Academy of Sciences, al. Lotników 32/46, 02-668 Warsaw, Poland \\ ${ }^{c}$ Grenoble High Magnetic Field Laboratory, CNRS, BP 166, 38042 Grenoble Cedex 9, France
}

\begin{abstract}
The rate equation is used for description of photoluminescence dynamics after pulsed excitation of various states of quantum dots. The picosecond dynamics of averaged charge state of quantum dot is described. We compare our simulations with the experiment and come up with the conclusion that probability of carrier capture weakly depends on quantum dot charge state and that electrons and holes are captured non-synchronously.
\end{abstract}

PACS numbers: 78.55.Et, 73.21.La

\section{Introduction}

Perspectives of application in quantum computation and quantum cryptography make semiconductor quantum dots an attractive research field. Quantum dots (QDs) might be used to obtain and manipulate qubits and their entangled states. Also one of the possibilities is to use quantum dots as sources of single photons-on-demand. In view of these perspectives, precise knowledge of the dynamics of electronic states in the quantum dot is of particular importance. In this work we use a rate equation model to describe the dynamics of photoluminescence from quantum dots excited by pairs of pulses with controlled separation between them. In particular, picosecond-scale dynamics of the QD charge state is analyzed.

Our goal was to describe an experiment which was done on typical CdTe-based QD sample [1]. The MBE grown sample contained self-organized $\mathrm{CdTe} / \mathrm{ZnTe}$ quantum dots with density $\approx 5 \times 10^{9} \mathrm{~cm}^{-2}$. It was excited by pairs of light pulses arriving with controlled temporal separation. The wavelength of the laser light was $400 \mathrm{~nm}$. The laser spot diameter was $\approx 0.5 \mu \mathrm{m}$. We measured the temporal integrated photoluminescence of selected quantum dot states as a function of delay between pulses (see Fig. 2a-c). More information about the experiment can be found in [2].

\section{Model}

We use the rate equation model to describe the process of non-resonant pulsed excitation of QDs. The energy of photons exciting the sample is above the energy gap of
ZnTe, so the electron-hole pairs are created in the barrier. Then both kinds of carriers are trapped separately into QDs, where they recombine producing photoluminescence.

So far, a rate equation model was successfully applied to simulate the results of single photon correlations as well as photoluminescence from exciton (X), charged exciton (CX) and double exciton (XX) states as a function of excitation power $[2,3]$. In this work we extend this model to include the results of double-pulse excitation. We modified the rate equations from Ref. [2] to incorporate more excitonic complexes identified in the PL spectrum [4].

\section{Results}

The considered states and transitions with related parameters are presented in Fig. 1. Parameters $\tau$ stand for the radiative lifetimes of QD states measured in the experiment. Following Ref. [2], $\alpha(\beta)$ corresponds to the probability of electron (hole) capture. We accounted for influence of residual carriers to the trapping rate by introducing $\gamma$ parameter as shown in Fig. 1. It reflects the fact that the long-range Coulomb interaction between the carrier(s) residing in the $\mathrm{QD}$ and a free carrier in the barrier should influence the trapping probability of the carrier by the QD.

From the measurements of the average QD charge state (see Fig. 2) we deduce that parameters $\alpha, \beta$ must be of the same order of magnitude. The actual capture rate is time-dependent when the excitation is pulsed. The main results of simulation were insensitive for exact temporal profile of carrier capturing. In presented results, this time-dependent capture rates were assumed to be in the 


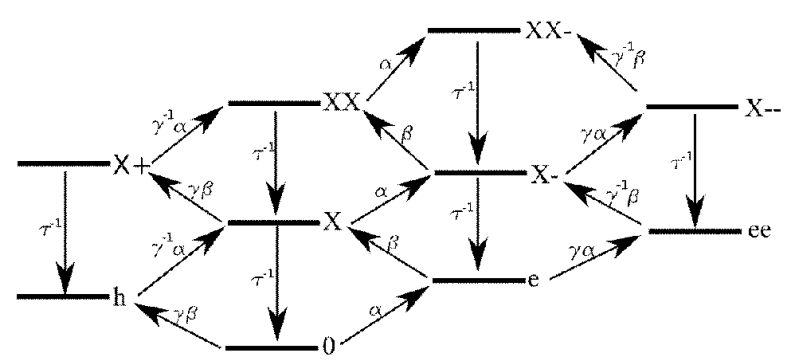

Fig. 1. QD states and transition considered in the model with related rate coefficients.

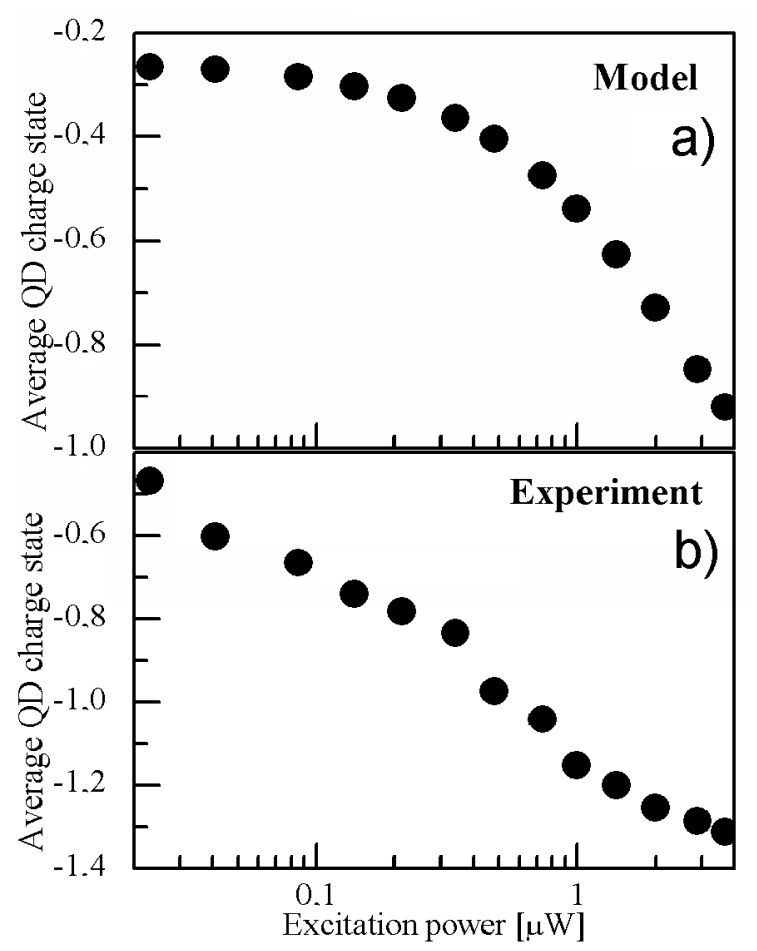

Fig. 2. QD charge state as a function of excitation power: model (top) and experiment (bottom).

form of Gaussian pulses with amplitudes proportional to the excitation power $P$.

For example, the electron capture rate in case of pair of excitation pulses separated by $\Delta t$ interval reads

$$
R_{0 e}(t)=P \alpha\left[\exp \left(\frac{-t^{2}}{2 \sigma}\right)+\exp \left(\frac{-(t-\Delta t)^{2}}{2 \sigma}\right)\right] .
$$

The rate equations are the following:

$$
\frac{\mathrm{d} \boldsymbol{p}}{\mathrm{d} t}=A \boldsymbol{p},
$$

where $A$ is the transition rate matrix and vector $\boldsymbol{p}$ describes the occupation probability. We took the ten-dimensional space of QD states as shown in Fig. 1. At given excitation power, a stationary state was computed by solving the equations numerically. Then time-integrated photoluminescence was computed for differ-

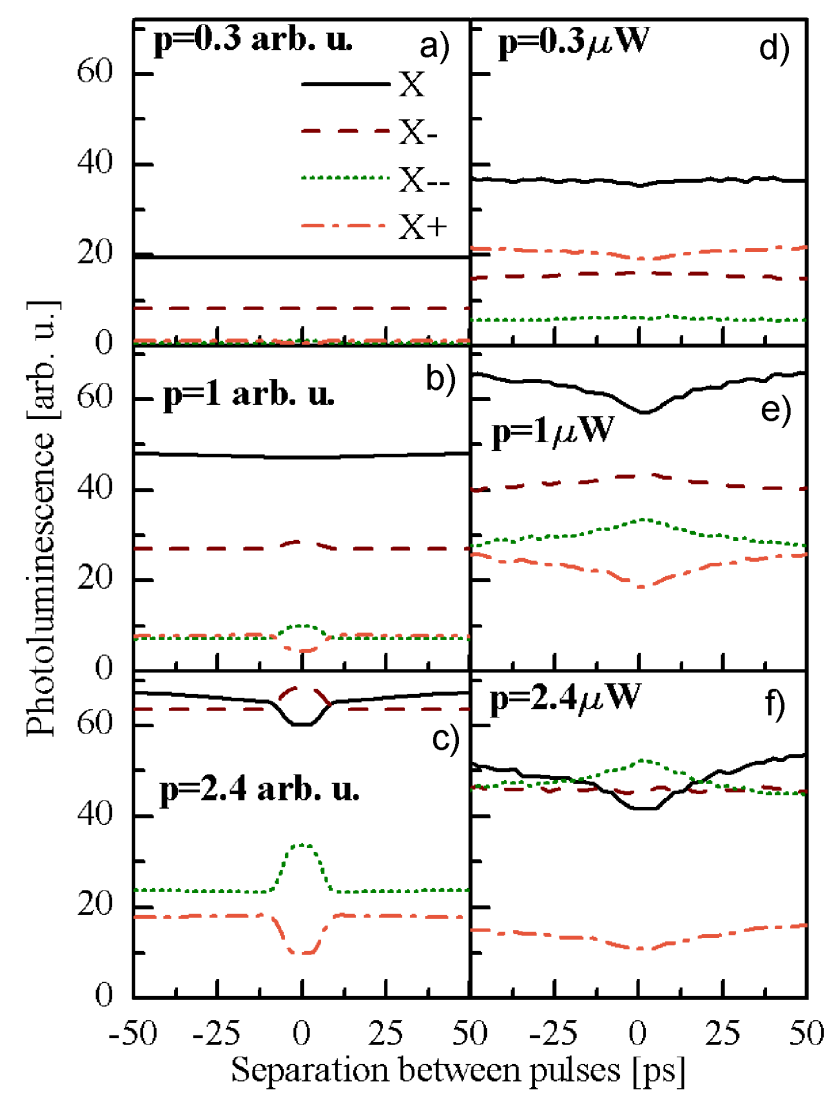

Fig. 3. Time-integrated luminescence intensity versus time delay between pulses for three different laser powers $p$ : model calculation (a)-(c) and experimental results (d)-(f).

ent values of temporal separation between the pulses in each pair, according to the formula

$$
I_{i}=\int p_{i} \mathrm{~d} t, \quad \text { where } i=\mathrm{X}, \mathrm{XX}, \ldots
$$

Our calculation showed that there is no characteristic feature appearing for small values of the temporal delay if the shape of time-dependent capture probability is taken identical for electrons and holes. However, introducing a small delay ( $\delta=5 \mathrm{ps}$ ) between the electron and hole capture pulses was enough to reproduce the essential features of the experimental results. It is shown in Fig. $3 \mathrm{~d}-\mathrm{f}$. The delay $\delta$ turned out to be the essential parameter of the model. Therefore the hole capture rate was modified as follows:

$$
R_{0 \mathrm{~h}}(t)=\frac{\beta}{\alpha} R_{0 \mathrm{e}}(t-\delta) .
$$

The difference between the arrival time of electrons and holes may originate from the influence of trapping centers in the barrier, acting differently on both types of carriers. Presence of the sharp features in the simulated profiles was observed regardless of exact values of input parameters. Therefore we cannot claim that the used set of parameters is unique. The radiative decay times (not 
essential for the fast processes studied here) were known from independent photoluminescence (PL) decay measurements. Surprisingly the results correctly describing the experiment were obtained only for $\gamma$ parameter close to 1. This indicates small influence of the QD charge on the carrier capture probabilities, which means that the Coulomb interaction is negligible for the carrier trapping by the QD.

The main discrepancies between simulations and experimental data occur for states $\mathrm{X}^{--}$and $\mathrm{X}^{+}$(Fig. 3). By referring to the scheme in Fig. 1, we assume that the first one cannot accept any additional electrons while the other one cannot accept holes. That results in faster saturation of $\mathrm{X}^{--}$and $\mathrm{X}^{+}$states. The cut-off of higher states in positively charged $\mathrm{QD}$ is justified by small valence band offset in the CdTe/ZnTe system [5]. The presented model reproduces also another experimental feature related to QD charge state.

The simulation of average charge state of the QD was computed in the following way:

$$
\bar{q}=\frac{I_{\mathrm{X}^{+}}-I_{\mathrm{X}^{-}}-2 I_{\mathrm{X}^{--}}}{I_{\mathrm{X}}+I_{\mathrm{X}^{+}}+I_{\mathrm{X}^{-}}+I_{\mathrm{X}^{--}}}
$$

shows that with increasing power quantum dot becomes more negative. Thus the simulation reproduces qualitatively the experimental results, as shown in Fig. 3. A quantitative description of this dependence of the QD charge state as a function of excitation power would require further extension of the model.

\section{Conclusions}

We succeeded in modeling the fast excitation dynamic of neutral and charged exciton states in $\mathrm{CdTe} / \mathrm{ZnTe} \mathrm{QDs}$ and managed to reproduce qualitatively essential features of experimental profiles. To achieve this goal we had to assume a non-synchronous capture of electrons and holes. It also turned out that the carrier capture probabilities weakly depend on the QD charge state.

\section{Acknowledgments}

This work has been partially supported by the Ministry of Science and Higher Education under research contracts financed in years 2006-2010.

\section{References}

[1] P. Wojnar, J. Suffczyński, K. Kowalik, A. Golnik, M. Aleszkiewicz, G. Karczewski, J. Kossut, Nanotechnology 19, 235403 (2008).

[2] J. Suffczyński, T. Kazimierczuk, M. Goryca, B. Piechal, A. Trajnerowicz, K. Kowalik, P. Kossacki, A. Golnik, K. Korona, M. Nawrocki, J. Gaj, Phys. Rev. B 74, 085319 (2006).

[3] T. Kazimierczuk, J. Suffczyński, A. Golnik, J. Gaj, P. Kossacki, Phys. Rev. B 79, 153301 (2009).

[4] T. Kazimierczuk, A. Golnik, M. Goryca, P. Wojnar, J. Gaj, P. Kossacki, Acta Phys. Pol. A 116, 882 (2009).

[5] H. Mathieu, J. Allegre, A. Chatt, P. Lefebvre, Phys. Rev. B 38, 7740 (1988). 